\title{
A case of I gG4-related interstitial lung disease showing usual interstitial pneumonia pattern: Unusual case for histological features with pathological proof
}

\author{
Seidai Sato ${ }^{1}$, Masaki Hanibuchi ${ }^{1}$, Makoto Tobiume $^{2}$, J un Kishi ${ }^{1}$, Yuko Toyoda ${ }^{1}$, Hiroshi Kawano ${ }^{1}$, \\ Mikiko Takahashi ${ }^{3}$, Yuh Fukuda ${ }^{3,4}$, Yasuhiko Nishioka ${ }^{1}$ \\ 1. Department of Respiratory Medicine and Rheumatology, Institute of Health Biosciences, The University of Tokushima \\ Graduate School, Tokushima, Japan. 2. Department of Internal Medicine, Kochi Red Cross Hospital, Kochi, Japan. \\ 3. Department of Analytic Human Pathology, Nippon Medical School, Tokyo, Japan. 4. Division of Diagnostic Pathology, \\ Itabashi Chuo Medical Center, Tokyo, Japan.
}

Correspondence: Yasuhiko Nishioka, M.D., Ph.D. Address: Department of Respiratory Medicine and Rheumatology, Institute of Health Biosciences, The University of Tokushima Graduate School, 3-18-15 Kuramoto-cho, Tokushima 770-8503, Japan. E-mail: yasuhiko@tokushima-u.ac.jp

Received: J une 27,2014

DOI : $10.5430 /$ crcp.v2n1p6
Accepted: July 23, 2014

URL: http://dx.doi.org/10.5430/crcp.v2n1p6

\section{Abstract}

The case was a 71-years-old male with chest abnormal shadow and persistent dry cough. Chest CT showed ground-glass opacities, traction bronchiectasis, and honeycomb-like change in the bilateral lower lobes. Laboratory tests revealed high serum IgG4 concentration. Pathological examination of the video-assisted thoracoscopic surgery biopsy taken from the right lung showed a pattern compatible with usual interstitial pneumonia (UIP) with strong infiltration of IgG4-positive plasma cells. Based on these findings, diagnosis of IgG4-related interstitial lung disease (ILD) was made. One year later, when ILD was progressed with the appearance of swelling of both submandibular glands, treatment with prednisolone (PSL) was started. When the dose of PSL was tapered to $7.5 \mathrm{mg}$, left pleural effusion emerged in chest CT. Further examination yielded the diagnosis of lung adenocarcinoma and he was treated with chemotherapy. After two cycles of chemotherapy, IgG4-related interstitial lung disease exacerbated, thus he was re-treated with moderate dose of PSL. Response to PSL treatment was good, but unfortunately, chemotherapy for lung cancer was discontinued due to the risk for re-exacerbation of ILD. To our knowledge, this is the eighth case of IgG4-related ILD ever reported and the second case diagnosed as UIP pattern pathologically.

\section{Key words}

IgG4, Interstitial lung diseases, Usual interstitial pneumonia, Lung cancer

\section{I ntroduction}

IgG4-related disease (IgG4-RD) is a newly recognized fibroinflammatory condition characterized by a dense lymphoplasmacytic infiltrate rich in IgG4-positive plasma cells into multiple organs, and often but not always, elevated serum IgG4 concentrations ${ }^{[1]}$. IgG4-RD has been described in virtually every organ system: the biliary tree, salivary glands, 
periorbital tissues, kidneys, lungs, lymph nodes, meninges, aorta, breast, prostate, thyroid, pericardium, and skin ${ }^{[1]}$. Although lung lesions in the IgG4-RD have been usually recognized for manifesting as interstitial pneumonia or inflammatory pseudo-tumor ${ }^{[2-4]}$, the clinical features of IgG4-related lung diseases were not fully elucidated. In recent years, seven cases of IgG4-related interstitial lung disease (ILD) with pathological confirmation had been reported ${ }^{[2,5-7]}$. Of these seven cases, six cases were pathologically diagnosed as non-specific interstitial pneumonia (NSIP) pattern, indicating that usual interstitial pneumonia (UIP) pattern was a rare manifestation in IgG4-related ILD.

In the present report, we describe a rare case of IgG4-related ILD that was pathologically confirmed as UIP pattern.

\section{Case presentation}

A 71-years-old male with diabetes mellitus was referred to our hospital for further examination of chest abnormal shadow (see Figure 1) and persistent dry cough. He was a current smoker (30 cigarettes/day for 50 years), but had no obvious history of exposure to any dust.

Figure 1. Chest X-ray film obtained upon admission in December 2008 shows volume loss and reticular shadows in both lower lungs.

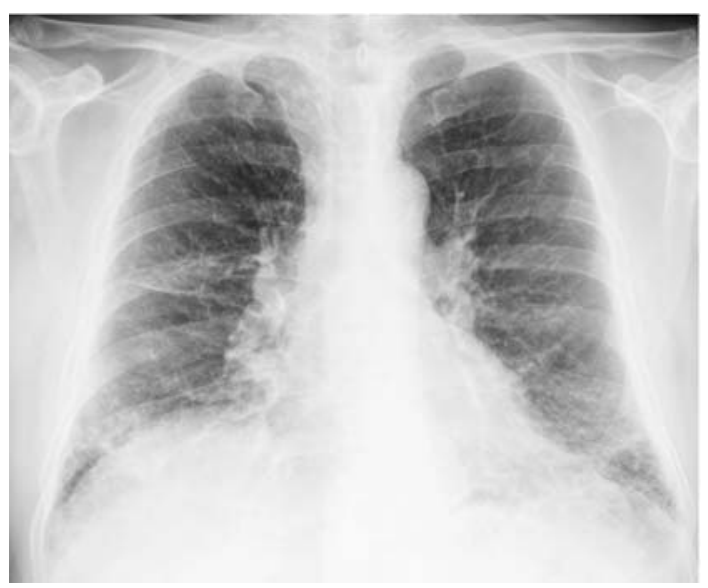

Physical examination revealed bilateral middle-to-late inspiratory fine crackles in the lower lung field, and finger clubbing. The percutaneous oxygen saturation in room air was $94 \%$. Chest CT showed the enlargement of axillary and mediastinal lymph nodes, ground-glass opacities, traction bronchiectasis, and honeycomb-like change especially in the bilateral lower lobes (see Figure 2). PET-CT revealed a large number of FDG-positive lymph nodes on both side of neck, parotid gland, bilateral hilar, axillary regions, and mediastinum. The results of pulmonary function tests are as follows; a vital capacity (VC) of 3.03 L (95.6\% predicted), forced expiratory volume in one second (FEV1.0) of $2.17 \mathrm{~L}$ (69.8\% predicted), and mildly reduced diffusing capacity (DLCO) of $7.15 \mathrm{ml} / \mathrm{min} / \mathrm{mmHg}(43.2 \%$ predicted).

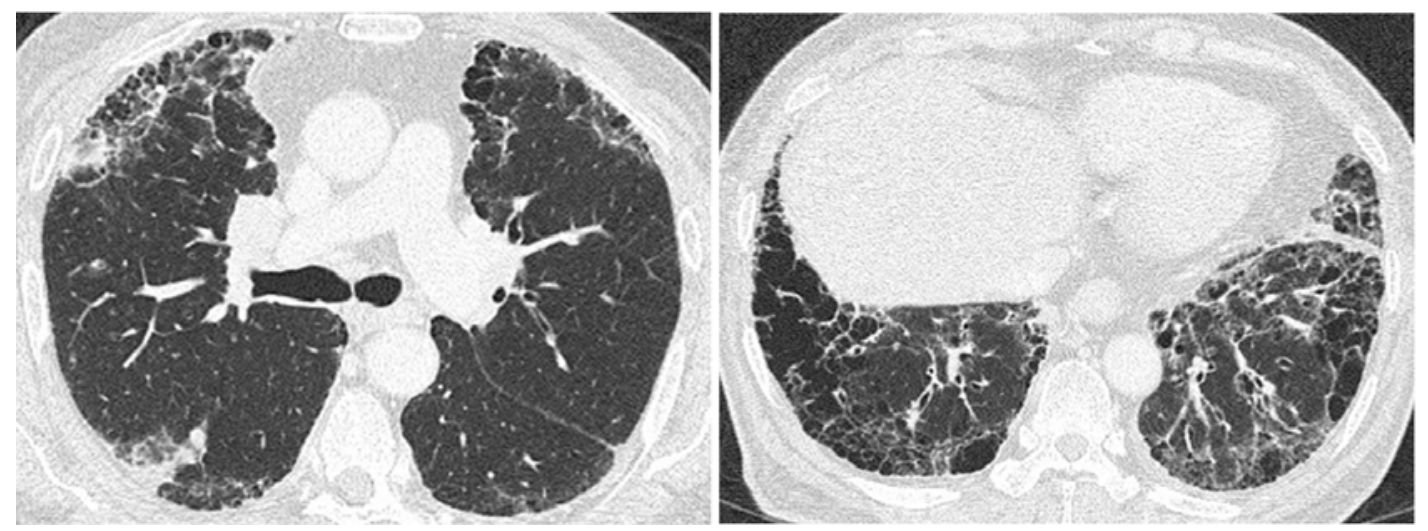

Figure 2. Chest CT shows pale ground-glass opacities, traction bronchiectasis, and honeycomb-like change especially in the bilateral lower lobes pleural side advantage. 
Peripheral blood examination was as follows: C-reactive protein was $0.27 \mathrm{mg} / \mathrm{dl}$. Serum electrolytes, renal function and liver function were normal. Notable laboratory values included: serum KL-6 $307 \mathrm{IU} / \mathrm{ml}$ (normal: < 500 U/ml), SP-D $207.3 \mathrm{ng} / \mathrm{ml}$ (normal: < $110.0 \mathrm{ng} / \mathrm{ml}$ ), SP-A $61 \mathrm{ng} / \mathrm{ml}$ (normal: < $43.8 \mathrm{ng} / \mathrm{ml}$ ), ACE $16.2 \mathrm{IU} / \mathrm{ml}$, TP $10.1 \mathrm{~g} / \mathrm{dl}$ (alb 34.4\%, $\alpha 1$-globlin $2.1 \%$, $\alpha 2$-globlin 8.2\%, $\beta$-globlin 4.9\%, $\gamma$-globlin 50.4\%), IgG 4,589 mg/dl, IgA $115 \mathrm{mg} / \mathrm{dl}, \operatorname{IgM} 20 \mathrm{mg} / \mathrm{dl}$, IgE $1054.6 \mathrm{IU} / \mathrm{ml}$, and IgG4 was increased to $2,970 \mathrm{mg} / \mathrm{dl}, 64.7 \%$ of total $\mathrm{IgG}$ (IgG4 normally comprises less than $6 \%$ of total $\mathrm{IgG})$. Anti-nuclear antibody was barely positive $(\times 40$ homogenous and speckled pattern). Perinuclear anti-neutrophil cytoplasmic antibody (ab), anti-RNP-ab, anti-SSA-ab, anti-SSB-ab, anti-Sm-ab, anti-Jo-1-ab, anti-Scl70-ab, anti-dsDNA-ab, and anti-centromere-ab, were negative.

Bronchoscopy with bronchoalveolar lavage was performed through the right B4 bronchus. The total cell number was $6.25 \times 105$ cells $/ \mathrm{ml}$ (macrophages $66.5 \%$, lymphocytes $19.1 \%$, neutrophils $0.2 \%$, eosinophils $14.3 \%$ ) and the CD4/8 ratio was 1.37. By transbronchial lung biopsy, neither malignant nor granulomatous lesions were observed. The result of Saxon test was within normal limits. The patients did not meet the criteria for a diagnosis of any collagen disease.
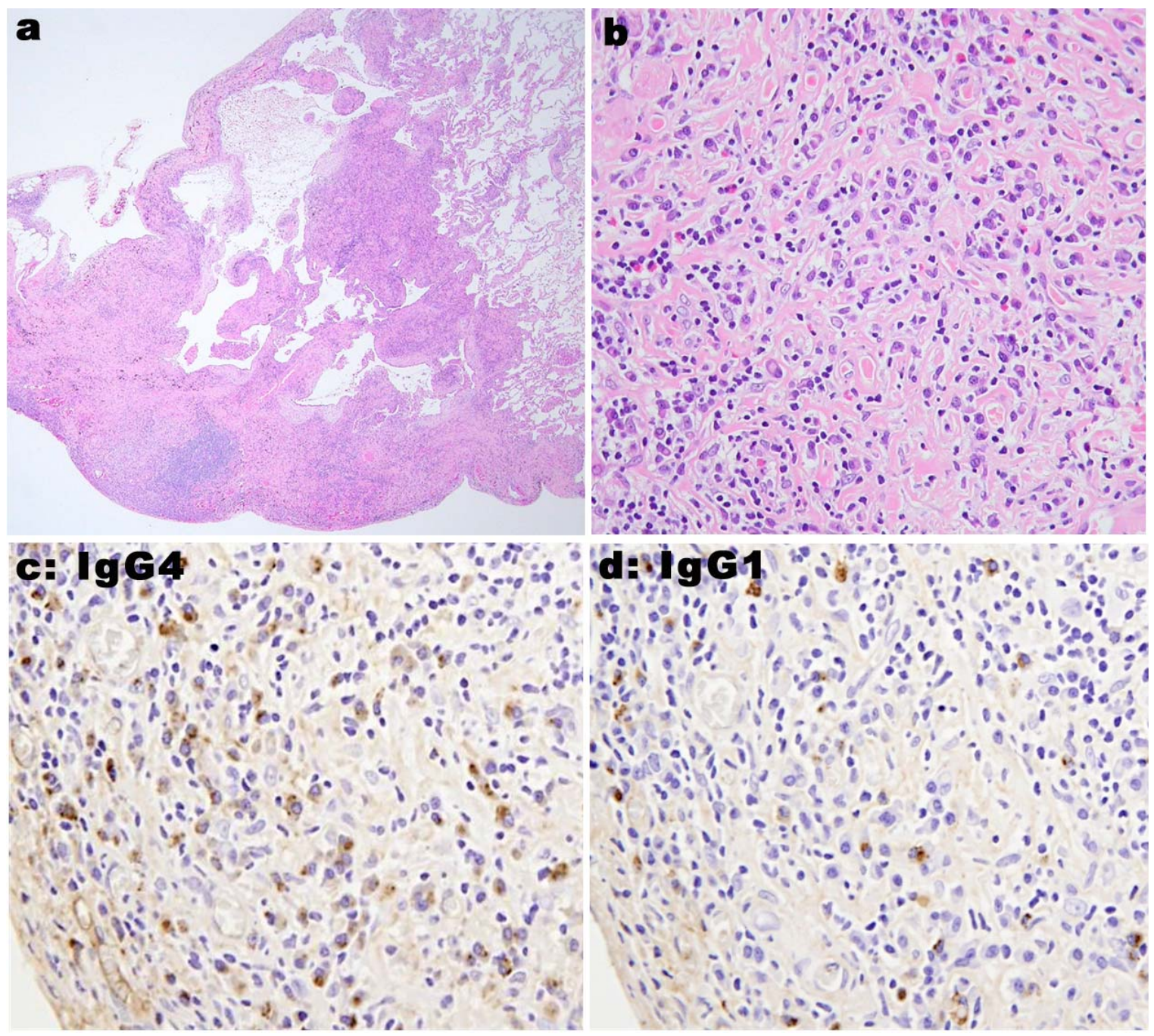

Figure 3. a, b: Histological findings of video-assisted thoracoscopic surgery biopsy specimen from right lower lung. Honeycomb lung structure $\left(^{*}\right)$ is detected, and fibroblastic foci are definite (arrows). Pleura shows thickening with inflammatory and fibrous granulation (a). Numerous plasma cells, lymphocytes and some eosinophils infiltration to the pleura and alveolar interstitium are observed (b). c, d: Immunostaining shows the infiltrates of IgG4-positive plasma cells in the interstitium. The mean number of IgG4-positive plasma cells (c) is about four times more than IgG1-positive plasma cells (d). 
To assist with diagnosis and its pathogenesis, video-assisted thoracoscopic surgery (VATS) of the right lower lung and superior mediastinal lymph nodes were performed in October 2009. Pathological specimens showed a pattern compatible with UIP with many plasma cells infiltration. Like moderate honeycomb changes, significant diffuse interstitial and pleural fibrosis was observed with fibroblastic foci on pathologic findings (see Figure 3a, 3b). There was no evidence of vasculitis and granulomatous lesions. Immunostaining showed infiltrates of IgG4-positive plasma cells in the interstitium. The mean number of IgG4-positive plasma cells was four times more than IgG1-positive plasma cells (see Figure 3c, 3d). Based on these findings, diagnosis of IgG4-related ILD was made. As ground-glass opacities in lung field ameliorated spontaneously and the patient did not exhibit any symptoms, he was followed up without any medication.

In October 2010, he became aware of a dry cough and shortness of breath upon exertion. In addition to respiratory symptoms, symmetric swelling on both submandibular glands was found by physical examination. Chest CT has been shown that the ground-glass opacity appeared again extensively, and DLCO in the respiratory function test was reduced significantly to $5.20 \mathrm{ml} / \mathrm{min} / \mathrm{mmHg}$ (32.2\% predicted). From these findings, he was diagnosed as the exacerbation of IgG4-related ILD. Moderate dose of prednisolone (PSL; $35 \mathrm{mg}, 0.5 \mathrm{mg} / \mathrm{kg} /$ day) therapy was started in November 2010, and his symptoms and laboratory findings were improved. Serum IgG was normalized ( $993 \mathrm{mg} / \mathrm{dl})$ and SP-D decreased gradually. Chest X-ray and chest CT findings as well as pulmonary function test demonstrated significant improvements. Dose of PSL was tapered gradually with good clinical course.

When the dose of PSL was tapered to $7.5 \mathrm{mg}$, chest CT showed no exacerbation of IgG4-related ILD, but revealed the presence of left pleural effusion. Cytopathologic examination of pleural fluid yielded the diagnosis of lung adenocarcinoma without EGFR mutation, then treatment with Carboplatin and Paclitaxel was started in March 2012. After 2 cycles of chemotherapy, IgG4-related ILD again exacerbated with diffuse ground-glass opacities in chest CT, thus he was re-treated with moderate dose of PSL. Response to PSL treatment was good, but unfortunately, chemotherapy for lung cancer was discontinued due to the risk for re-exacerbation of ILD.

\section{Discussion}

Recently, increasing attention has been paid for IgG4-RD in various fields. IgG4-RD was firstly reported with regard to autoimmune pancreatitis (AIP) in $2001^{[8]}$. In this report, the authors suggested that increasing IgG4 is associated with pathology in AIP, and measuring IgG4 was a useful means of distinguishing this disorder. Later, Hamano et al. reported that IgG4-positive plasma cell infiltration was observed characteristically in retroperitoneal fibrosis and tissue lesions of the pancreas ${ }^{[9]}$.

Accumulating recent evidences revealed that similar pathological change also occurs in multiple organs, including the lungs ${ }^{[10]}$. Lung lesions in the IgG4-RD have been usually recognized for manifesting as interstitial pneumonia or inflammatory pseudo-tumor ${ }^{[2-4]}$. However, there are few case reports regarding IgG4-related lung diseases at present, and the clinical features of IgG4-RD in the lungs are not fully elucidated. Moreover, cases with pathological confirmation of IgG4-positive cell infiltration in the lungs were even less.

The present case indicates three interesting points regarding IgG4-related ILD. The first point is that the histological pattern of ILD was UIP. In recent years, seven cases of IgG4-related ILD with pathological confirmation had been reported ${ }^{[2,5-7]}$. Of these seven cases, six cases are diagnosed as NSIP pattern pathologically, and only one case was a UIP pattern. In the only reported IgG4-ILD case that was diagnosed as UIP pattern, chest CT findings were strikingly different from the radiologic pattern expected in idiopathic pulmonary fibrosis (IPF) ${ }^{[7]}$. Unlike this case, chest CT findings were relatively compatible with radiologic pattern of IPF in our case. But as with the previous case, serum IgG4 concentration and radiologic features met all diagnostic criteria recently proposed for IgG4-related disease, which led to the definite diagnosis of IgG4-related ILD ${ }^{[11]}$. In addition, the appearance of the bilateral swelling of submandibular glands one year 
after VATS and the rapid and significant response to steroid treatment supported this diagnosis. Serum IgG4 was also decreased with the treatment, indicating that IgG4 might be a good biomarker to assess disease activity.

The second useful information could be that there is a case of ILD preceding the appearance of typical IgG4-RD such as AIP as well as Mikulicz's disease like the present case. Similar to the cases reported before ${ }^{[2,5,7]}$. AIP and Mikulicz's disease was not clinically apparent in the present case. However, swelling of the salivary glands was observed one year after VATS diagnosis of ILD in this case. As IgG4-positive plasma cell infiltration into salivary glands was observed by histology, the present case was considered to be complicated by Mikulicz's disease during the disease progression, but not at the first visit.

Third point is in the relation of IgG4-RD and malignancy. Recently, Yamamoto et al. analyzed the relationship between IgG4-RD and malignancies ${ }^{[12]}$. They reported that malignant disease including lung cancer, colon cancer, and lymphoma has occurred in 11 cases out of 106 cases of IgG4-RD (10.4\%) and that the standardized incidence ratio for these malignancies in IgG4-RD was 383.0, which was higher than that for the general population. These observations emphasized the importance of the systemic screening of the possible existence of malignancies in patients with IgG4-RD at the time of diagnosis and during follow-up care. The present case was also complicated by lung cancer (adenocarcinoma) during his clinical course. While IgG4-related ILD ameliorated at the time of diagnosis of lung cancer, it has again deteriorated as possibly triggered by the chemotherapy for lung cancer, which resulted in the cessation of lung cancer treatment. These observations suggested that the complication of lung cancer with IgG4-related ILD might lead to unfavorable outcomes because of the difficulties of adequate medical treatment. The present case has progressed at an advanced stage of lung cancer when diagnosed. Adžić et al. reported that majority of patients who complicated with lung cancer in connective tissue disease (CTD) also presented at an advanced stage of lung cancer with $87 \%$ having stage III or IV disease ${ }^{[13]}$. And they also proposed that the necessity of making efforts for earlier recognition of lung cancer in patients with CTD with regular follow-up, including chest imaging. Current investigations in oncogenesis and stimulations of fibrogenous processes will contribute to our better understanding of the pathogenesis of lung cancer and $\mathrm{CTD}^{[14]}$. It may be related to pathological of lung cancer complicated with IgG4-related ILD.

Although the diagnostic criteria of some IgG4-RDs, such as AIP and IgG4-related Mikulicz's disease, have been established, the diagnostic criteria of IgG4-related ILD without systemic involvement have not been created yet due to little understanding of its clinical features and laboratory and radiologic findings. Further studies addressing the evaluation of IgG4 concentration in the lung and serum in patients with ILD may shed further light on the association of IgG4 with ILD and the underlying pathophysiology.

In conclusion, we reported a rare case of IgG4-related ILD that was pathologically confirmed as UIP pattern, preceding the appearance of typical IgG4-RD. To the best of our knowledge, this is the eighth case of IgG4-related ILD ever reported and the second case pathologically diagnosed as UIP pattern. Despite its histological pattern as UIP, a favorable response was obtained by steroid treatment, suggesting the therapeutic efficacy of moderate dose of PSL. Moreover, the complication of lung cancer emphasized the importance of the systemic screening of the possible existence of malignancies in patients with IgG4-RD.

\section{Conflict of interests}

The authors declare that they have no conflict of interests.

\section{References}

[1] Stone JH, Zen Y, Deshpande V. IgG4-related disease. N Engl J Med. 2012 Feb 9; 366(6): 539-51. PMid: 22316447. http://dx.doi.org/10.1056/NEJMra1 104650 
[2] Takato H, Yasui M, Ichikawa Y, Fujimura M, Nakao S, Zen Y, et al. Nonspecific interstitial pneumonia with abundant IgG4-positive cells infiltration, which was thought as pulmonary involvement of IgG4-related autoimmune disease. Intern Med. 2008; 47(4): 291-4. PMid: 18277032. http://dx.doi.org/10.2169/internalmedicine.47.0411

[3] Taniguchi T, Ko M, Seko S, Nishida O, Inoue F, Kobayashi H, et al. Interstitial pneumonia associated with autoimmune pancreatitis. Gut. 2004 May; 53(5): 770. PMid: 15082601.

[4] Zen Y, Kitagawa S, Minato H, Kurumaya H, Katayanagi K, Masuda S, et al. IgG4-positive plasma cells in inflammatory pseudotumor (plasma cell granuloma) of the lung. Hum Pathol. 2005 Jul; 36(7): 710-7. PMid: 16084938. http://dx.doi.org/10.1016/j.humpath.2005.05.011

[5] Tanaka K, Nagata K, Tomii K, Imai Y. A case of isolated IgG4-related interstitial pneumonia: a new consideration for the cause of idiopathic nonspecific interstitial pneumonia. Chest. 2012 Jul; 142(1): 228-30. PMid: 22796844. http://dx.doi.org/10.1378/chest.11-1858

[6] Zhang H, Shi JH, Feng RE, Tian XL, Xu ZJ, Xun WB, et al. The clinicopathological analysis of 4 cases of IgG4-related nonspecific interstitial pneumonia. Zhonghua Jie He He Hu Xi Za Zhi. 2012 Oct; 35(10): 747-51. PMid: 23289991.

[7] Wibmer T, Kropf-Sanchen C, Rüdiger S, Blanta I, Stoiber KM, Rottbauer W, et al. Isolated IgG4-related interstitial lung disease: unusual histological and radiological features of a pathologically proven case. Multidiscip Respir. 2013 Mar 19; 8(1): 22. PMid: 23509921. http://dx.doi.org/10.1186/2049-6958-8-22

[8] Hamano H, Kawa S, Horiuchi A, Unno H, Furuya N, Akamatsu T, et al. High serum IgG4 concentrations in patients with sclerosing pancreatitis. N Engl J Med. 2001 Mar 8; 344(10): 732-8. PMid: 11236777. http://dx.doi.org/10.1056/NEJM200103083441005

[9] Hamano H, Kawa S, Ochi Y, Unno H, Shiba N, Wajiki M, et al. Hydronephrosis associated with retroperitoneal fibrosis and sclerosing pancreatitis. Lancet. 2002 Apr 20; 359(9315): 1403-4. http://dx.doi.org/10.1016/S0140-6736(02)08359-9

[10] Neild GH, Rodriguez-Justo M, Wall C, Connolly JO. Hyper-IgG4 disease: report and characterisation of a new disease. BMC Med. 2006 Oct 6; 4: 23. PMid: 17026742. http://dx.doi.org/10.1186/1741-7015-4-23

[11] Okazaki K, Umehara H. Are classification criteria for IgG4-RD now possible? The concept of IgG4-related disease and proposal of comprehensive diagnostic criteria in Japan. Int J Rheumatol. 2012; 2012: 357071.

[12] Yamamoto M, Takahashi H, Tabeya T, Suzuki C, Naishiro Y, Ishigami K, et al. Risk of malignancies in IgG4-related disease. Mod Rheumatol. 2012 Jun; 22(3): 414-8. PMid: 21894525. http://dx.doi.org/10.3109/s10165-011-0520-x

[13] Adzić TN, Pesut DP, Nagorni-Obradović LM, Stojsić JM, Vasiljević MD, Bouros D. Clinical features of lung cancer in patients with connective tissue diseases: a 10-year hospital based study. Respir Med. 2008 Apr; 102(4): 620-4. PMid: 18178071. http://dx.doi.org/10.1016/j.rmed.2007.10.022

[14] Bouros D, Hatzakis K, Labrakis H, Zeibecoglou K. Association of malignancy with diseases causing interstitial pulmonary changes. Chest. 2002 Apr; 121(4): 1278-89. PMid: 11948064. http://dx.doi.org/10.1378/chest.121.4.1278 